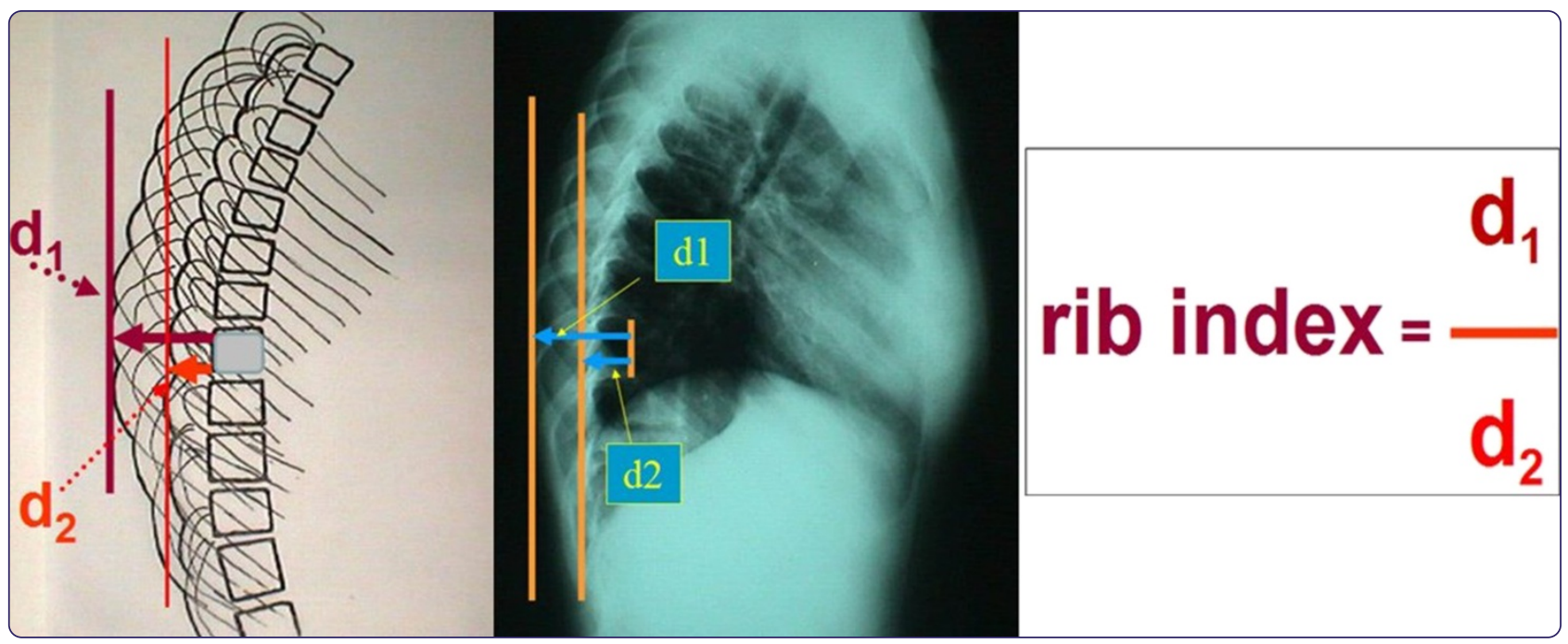

\title{
Rib index
}

\section{Grivas}




\title{
Rib index
}

\author{
Theodoros B Grivas
}

\begin{abstract}
This article analyzes the double rib contour sign (DRCS) and the rib index (RI). The analyzed topics are 1. the history of presentations - publication of DRCS-RI, 2. the study source origin: school screening for idiopathic scoliosis (IS), 3. what the DRCS and the RI are- Description, 4. the quantification of the DRCS - RI, 5. a reliability study for RI 6. how much the rib index is affected by the distance between the radiation source and the irradiated individual, 7 . the implications on IS aetiology, 8. the applications of Rib index for a. documentation of the deformity, $b$. assessment of physiotherapy, c. assessment of brace treatment and d. pre- and post-operative assessment; assessment of the rib-cage deformity correction on the transverse plane, 9. the use of RI and implications for screening policies 10. the reference of the RI method in spinal textbooks and finally 11. the citations in Google Scholar.
\end{abstract}

\section{The history of presentation and publication of DRCS-RI}

The DRCS and RI were initially presented in Greece during the $25^{\text {th }}$ "Nicolas Giannestras- Panayiotis Smyrnis" Anniversary Symposium of Spinal Column Diseases, at the Porto Rio Hotel, Patras, Greece, 21-23 May 1999 [1]. It was then presented in the International Research Society of Spinal Deformities meeting at Clermont Ferrand, Château du Marand, France, 23-26 May 2000 [2]. Subsequently, it was published in 2002 [3]. This publication focused on the implications of DRCS on the aetiology of idiopathic scoliosis [3].

\section{The study source origin: school screening for IS}

The scoliosis school screening program of the Orthopaedic Department of "Thriasio" General Hospital of Attica started in the school year of 1996. As far as we know, it has been the only program screening children attending the first grade of the primary school aged 5 to 6 years to the last grade of high school aged 17 to 18 years and therefore the collected data covered this wide range of ages. The screened asymmetric children of this program were referred to the scoliosis clinic of that department for further assessment. The children with a scoliometer reading more than 7 degrees were assessed radiographically. The thoracic cage and the spine were assessed for coronal (scoliosis) and sagittal (kyphosis-lordosis) Cobb angle and the segmental left and right RVAs of the rib-cage. In the

Correspondence: tgri69@otenet.gr

Orthopaedic \& Traumatology Department, "Tzaneio" General Hospital of Piraeus, Piraeus, Greece lateral spinal radiographs of all asymmetric children it was noticed that there was a double rib contour (DRC), an interesting radiologic sign not previously described in the available to us literature. We coined it "double rib contour sign" (DRCS).

\section{What are the DRCS and the RI - Description}

All lateral spinal radiographs in IS show a DRCS of the thoracic cage, a radiographic expression of the rib hump $(\mathrm{RH})$, Figure 1. The outline of the one hemi-thorax (convex) overlies the contour of the other hemi-thorax (concave). Then the rib index (RI) method extracted from the DRCS was introduced in order to quantify the severity of the double rib contour (DRC) that is to evaluate the rib hump deformity in IS patients in an attempt to create a safe reproducible way to assess the $\mathrm{RH}$ deformity based on lateral radiographs. This assessment actually represents the appraisal of the transverse plane rib-cage deformity, a method applied to the lateral spinal radiographs, which has not been presented earlier in the available to us literature, Figure 1.

\section{Quantification of the DRC sign}

For the quantification of the DRCS and formation of the RI we:

1. Determine the most extended rib point; $\mathrm{p} 1$. This is the osculation point of a vertical line tangential to the most extended point of the most extending rib contour (convex).

2. Draw a vertical line passing in p1; Line 1 . 


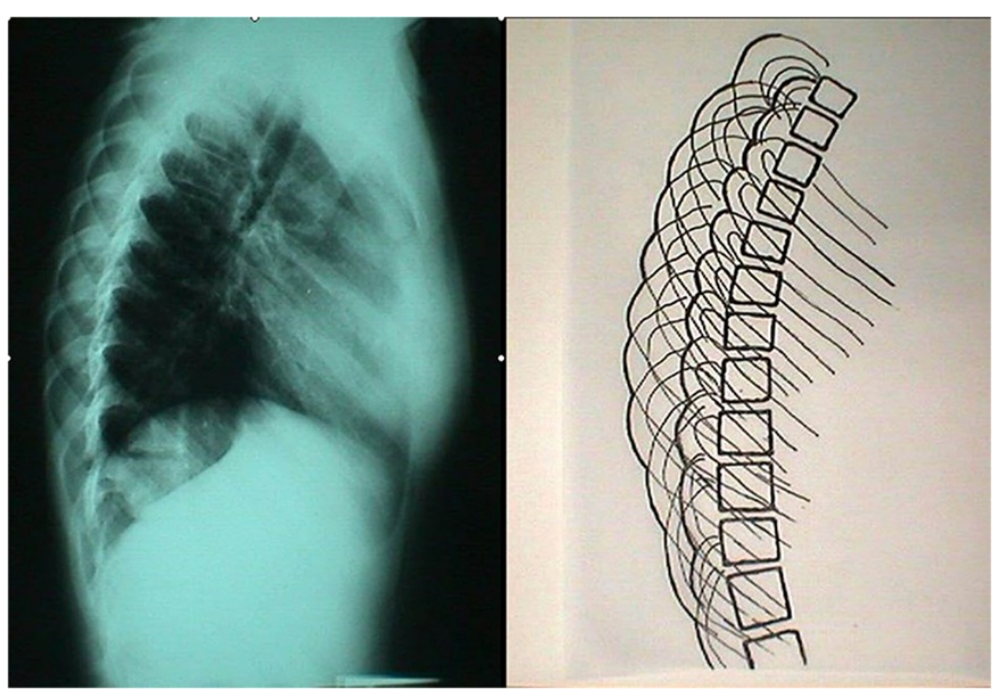

Figure 1 The double rib contour sign (DRCS) of the thoracic cage in a standing spinal radiograph.

3. Determine the least projected rib point; $\mathrm{p} 2$. This is the osculation point of a vertical line tangential to the most extended point of the least extending rib contour (concave).

4. Draw a vertical line passing in $\mathrm{p} 2$; Line 2.

5. Determine the corresponding vertebra and its posterior margin line; Line 3.

6. Measure the distance from line 3 to Line 1 and Line 2.

7. The distance between Line 3 and Line 1 is $\mathrm{d} 1$.

8. The distance between Line 3 and Line 2 is $\mathrm{d} 2$.

In the lateral radiographs, the posterior body's margin line of an un-rotated corresponding vertebra represents the Line 3. When the corresponding vertebra is rotated, the posterior margin of the vertebral body shows double line. Then the Line 3 is the parallel one that passes in the middle of this double line.
The corresponding vertebra is the one at the level of d1. We define this in order to draw the Line 3.

Therefore $\mathrm{d} 1$ is the distance between the most extended point of the most extending rib contour and the posterior margin of the corresponding vertebra on the lateral scoliosis films, while $\mathrm{d} 2$ is the distance from the least projected rib contour and the posterior margin of the same vertebra.

Lastly the "rib index" is defined as the $\mathrm{d} 1 / \mathrm{d} 2$ ratio (calculated by the ratio of spine distances $\mathrm{d} 1 / \mathrm{d} 2$ ). At a symmetric and non-deformed thorax the rib contour (RC) lines are practically superimposed and the "rib index" is $\approx 1$, Figure 2 .

\section{Reliability study}

The variations of the RI in a pair set of lateral chest radiographs in 49 randomized volunteers were estimated. Each of these subjects had two consequent chest lateral

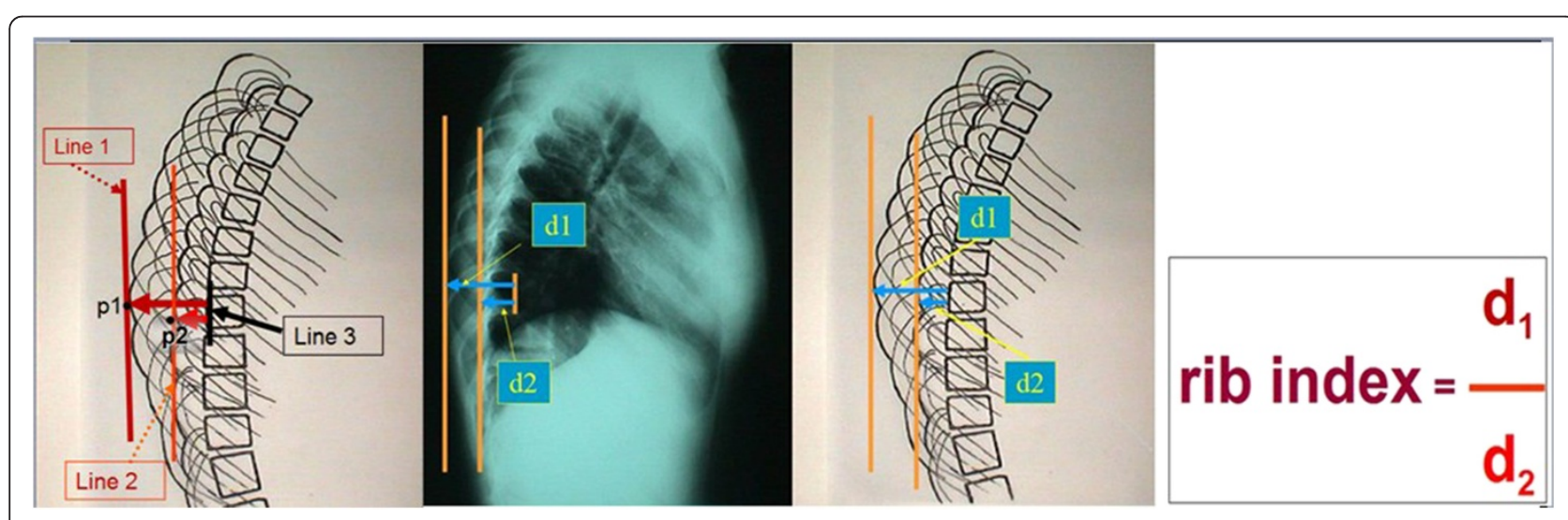

Figure 2 The Rib Index (RI) of the thoracic cage in a standing spinal radiograph. 
radiographs (LRs) at the radiological department of the hospital by the same technician during the course of treatment (named A and B group of LRs) in a standard position, Figure 3. The cassette was on the left side of the patient. The distance from the radiation source during lateral chest radiographs was chosen to be $1,80 \mathrm{~m}$, that is the one used in the radiographs for the scoliotic children according to the American College of Radiology's recommendations (2009) [4]. The RI was calculated in both LRs of each patient. The statistical analysis included the paired t-test in the set of LRs, its correlation coefficient, the intra- and inter-observer error using the formula $(\mathrm{SD} / \sqrt{ } 2) / 2$, where SD is this of the differences of the two sets of measurement (As-Bs). The SPSS v16 statistical package was used. It was found that in the 49 pairs of LRs there was no statistical difference of the RI, (paired t-test $\mathrm{p}<0.314$ ). The RI in the A and $\mathrm{B}$ group of LRs was highly correlated (correlation coefficient $\mathrm{R}=0,924, \mathrm{p}<0.0001$ ). The intra-observer error was 0.0080 , while the interobserver error was 0.0213 , in terms of $95 \%$ confidence interval (CI). Therefore the RI proves to be a reliable method to evaluate the thoracic deformity or the effect of surgical or non-operative treatment on the IS rib-cage deformity (hump). It was also demonstrated that the RI is a simple method and a safe reproducible way to assess the RH deformity based on lateral radiographs, without the need for any other special radiographs and exposure to additional radiation [5].

Ethical approval was provided for this study by the Ethical Committee of the "Attikon" General University Hospital of Athens, Greece". This study was in compliance with the Helsinki Declaration.

\section{How much is the RI affected by the distance between the radiation source and the irradiated individual?}

A validity study of DRCS was implemented to appraise how the RI is affected by the distance between the radiation source and the irradiated child.

The American College of Radiology's (2009) guidelines for obtaining radiographs for scoliosis in children recommend that the distance from the scoliotic child to the film is $1,80 \mathrm{~m}$ [4]. Normal values for the transverse diameter of the ribcage in children aged 6-12 years were considered those reported by Grivas in 1988 (Figures 4, 5 and 6) [6]. According to the Euclidean geometry (Figure 7), d1/d2 = 1.073 at a normal child 12 years of age, provided that the distance $\Delta \mathrm{Z} \approx 12 \mathrm{~cm}(11,84)$ and $\mathrm{EA}=180 \mathrm{~cm}$, with transverse rib-cage diameter of the child $22 \mathrm{~cm}$ (Figure 8 ). The values of the transverse rib-cage diameter measurements are presented in centimeters by age (2-12 years old) in boys (Figure 5 ) and in girls (Figure 6).

This validity study demonstrated that the DRCS is substantially true and practically the RI is not affected by the distance between the radiation source and the irradiated child [7].

However the positioning of the IS patient for the radiographic examination is very important. Each radiograph must be taken by technicians trained to do radiography in a standard way in any hospital or laboratory.

The $1,8 \mathrm{~m}$ distance between the radiation source and the irradiated individual may vary, depending on the examinee's body size. In order to eliminate this limitation, we calculated the $\mathrm{d} 1 / \mathrm{d} 2$ ratio using a couple of distances $(1,9 \mathrm{~m}$ and $1,7 \mathrm{~m})$ between the radiation source and the
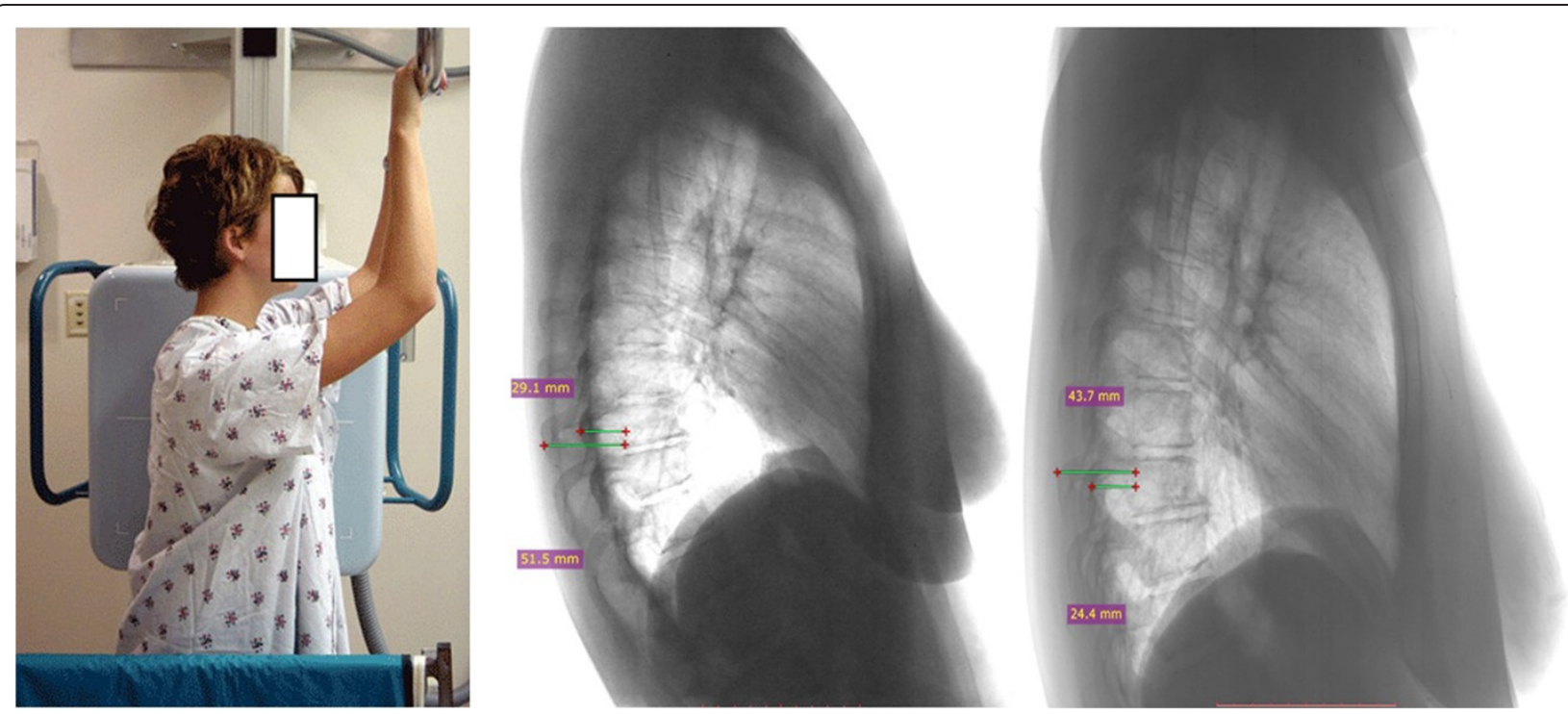

Figure 3 The radiographs were obtained in a standard position. Measurements in two radiographs. 


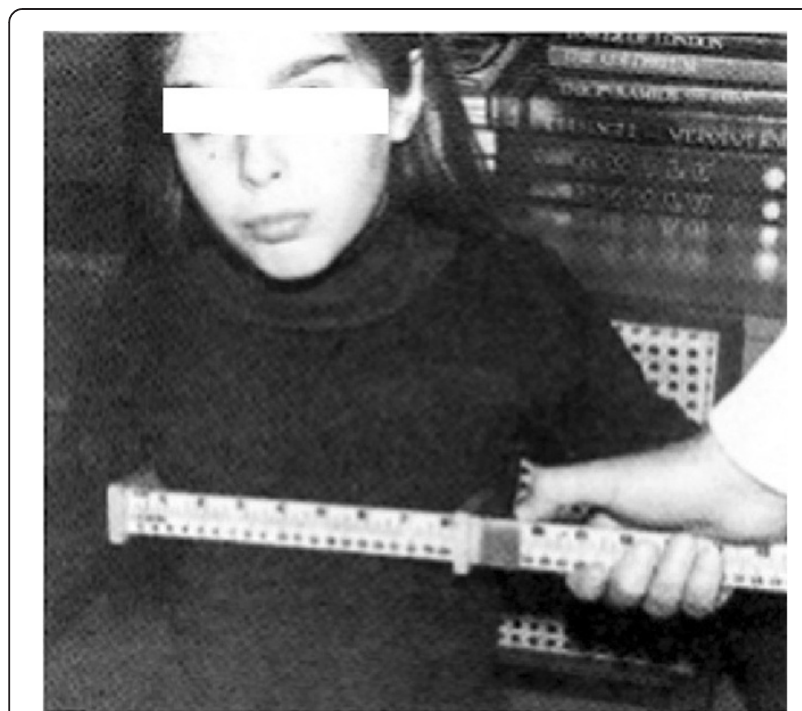

Figure $\mathbf{4}$ The way the transverse diameter of the rib-cage is documented in a child using a special anthropometric tool.

irradiated child. This variation corresponds to different body sizes $(1,9 \mathrm{~m}$ for larger/fatter and $1,7 \mathrm{~m}$ for smaller/ thinner children respectively). The results were almost similar and statistically insignificant. In conclusion, body size was found irrelevant in the measurement of the RI.

\section{Implications on aetiology of IS}

At the introduction it was noted that the screened asymmetric children of our screening program, with equal or more than 7 degrees of scoliometer reading, were referred to the scoliosis clinic for further assessment. In a cohort of 133 similar consequent children spinal postero-anterior and lateral radiographs were obtained, [1-3]. The DRCS was assessed in children with and without late onset idiopathic scoliosis (LOIS) with $10^{\circ}-20^{\circ} \mathrm{Cobb}$ angle and it was examined whether the deformity of the thorax or that of the spine develops first in IS. The cohort of children under investigation included 47 boys with a mean age 13.28 years and 86 girls with mean 13.39 years. All of them had a scoliometer reading (Angle of trunk rotation or inclination ATR or ATI) ATI $\geq 7^{\circ}$.

The Cobb angle was also assessed. Five groups of children were formed, as described below:

group 1 children with no scoliosis and straight spines $\mathrm{n}=27$ (12 boys 15 girls)

group 2 children with no scoliosis, spinal curvature having

a Cobb angle $1^{\circ}-9^{\circ}$

$\mathrm{n}=13$, ( 6 boys +7 girls $)$

group 3 children with thoracic scoliosis $10^{\circ}-20^{\circ}$

$\mathrm{n}=47$ (17 boys +30 girls)

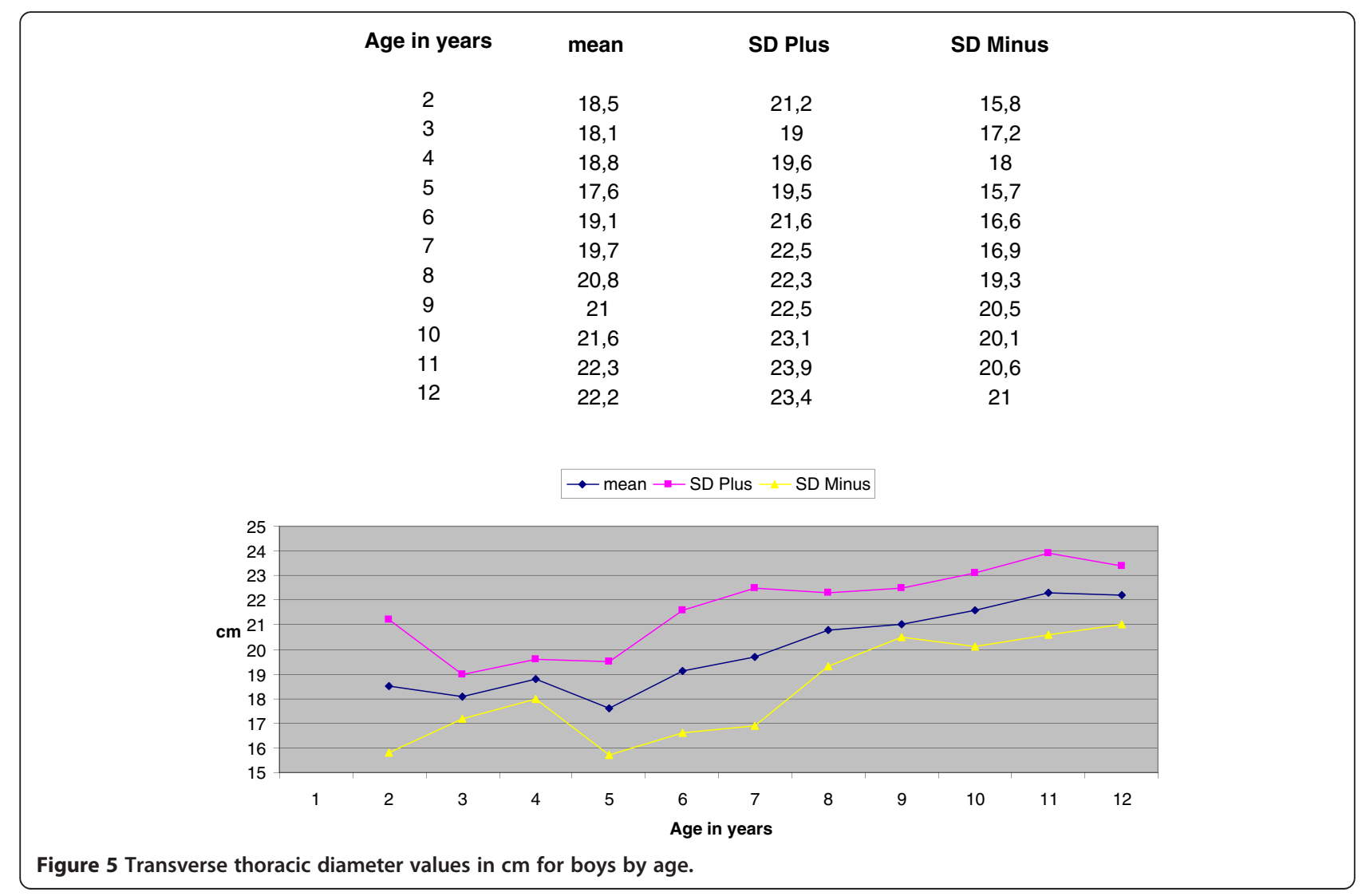


group 4 children with thoracolumbar scoliosis $10^{\circ}-20^{\circ}$ $\mathrm{n}=14$ ( 4 boys +10 girls)

group 5 children with lumbar scoliosis $10^{\circ}-20^{\circ}$

$\mathrm{n}=28$ (7 boys +21 girls)

The statistical analysis was done using the SPSS-PC v10 package using the following techniques: frequencies, descriptive (mean, range, min, max, sd dev, sd err, kurtosis, skewness), ANOVA, Kruskal-Wallis test, scatter plot, Pearson Correlation coefficient and independent Samples T-test. The mean RI in group 1 was 1.45 , in

\section{The triangles $\mathrm{EA} \Gamma$ and $\mathrm{EA} \Gamma$ ' are similar}

$\Rightarrow \frac{d_{1}}{d}=\frac{E A}{E \Delta} \Rightarrow d_{1}=d * \frac{E A}{E \Delta}$

The triangles EAB and EZB' are similar

$\Rightarrow \frac{d_{2}}{d}=\frac{E A}{E Z} \Rightarrow d_{2}=d * \frac{E A}{E Z}$

$\frac{(1)}{(2)}=\frac{d_{1}}{d_{2}}=\frac{d *(E A / E \Delta)}{d *(E A / E Z)}=\frac{E Z}{E \Delta}=\frac{E \Delta+\Delta Z}{E \Delta}=1+\frac{\Delta Z}{E \Delta}$

Figure 7 The Euclidean geometry used for the assessment of d1/d2 quotient. group $\mathbf{2}=1.51$, in group $3=1.56$, in group $\mathbf{4}=1.59$ and in group $5=1.47$. Interestingly, no RI sex differences for boys and girls were found and there was no correlation of the Cobb angle with the RI for the thoracic, thoracolumbar and lumbar mild LOIS groups (scoliosis $10^{\circ}-20^{\circ}$ Cobb angle). It was recognized that in all school-screening referrals (having ATI $\geq 7^{\circ}$ ), the thoracic deformity in terms of the DRCS has already been developed. It was also interesting to note that there were $20 \%$ of children with straight spines, $10 \%$ of children having curves with Cobb angle $1^{\circ}-9^{\circ}$, and $70 \%$ of children suffering IS. The non-

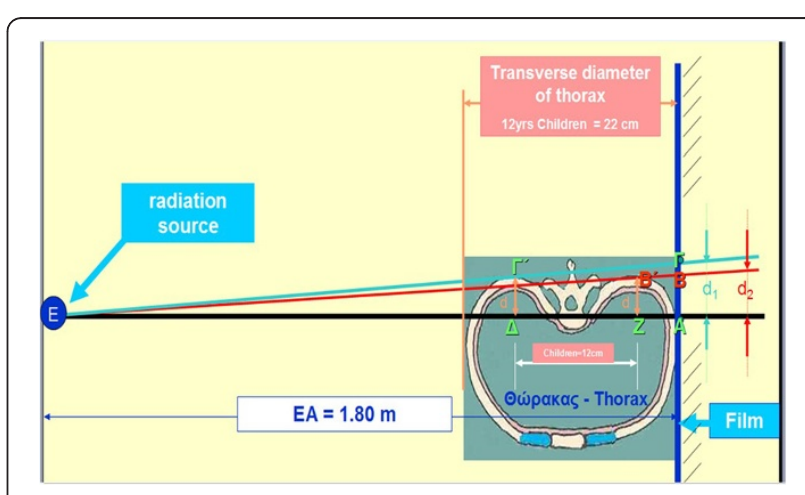

Figure 8 The set up for obtaining the radiograph and the used points (Greek letters) for the assessment of $\mathrm{d} 1 / \mathrm{d} 2$ quotient. 
scoliotics were followed due to the existing $\mathrm{RH}$; they were 1,5-2 years younger than the ones who had already developed IS and they had both a RI $\approx 1,5$. The DRCS was present in all referrals; in contrary there was no scoliotic spine without it, as the DRC sign is always present in scoliotic lateral spinal radiographs with no exception. This study led us to the conclusion that the DRCS is primarily the result of rib deformation and secondarily of vertebral rotation, because DRCS could be present in straight spines with no vertebral rotation. This observation supports the hypothesis that in IS the deformity of the thorax develops first and the deformity of the spine succeeds $[1-3,8]$.

Longitudinal follow-up results of data from school screening strengthen this statement [9].

\section{Applications of Rib index}

The RI can be applied for four purposes in the treatment of scoliosis. They are:

\section{a. Documentation of the deformity \\ b. Assessment of Physiotherapy \\ c. Assessment of brace treatment \\ d. Pre-and Postoperative assessment}

\section{Documentation of the deformity}

Using the RI we can assess and document the transverse plane thoracic deformity from lateral spinal radiographs.

\section{Assessment of Physiotherapy}

The RI, as an objective measure to document changes of the RH deformity, was used in non-operative treatment of IS using specific scoliosis physiotherapeutic exercises, (SSPE). Lebel and Lebel reported on a Risser 4 progressive AIS patient treated with the Schroth Method [10].

The authors reported: At diagnosis, the mean RI was 1.658 and the Cobb angle was $45^{\circ}$. In August 2012, the mean RI and Cobb angle increased to 2.352 and $56^{\circ}$ respectively, indicating an increase in $\mathrm{RH}$ deformity and progression of scoliosis. In November 2012, after 12 weeks of SSPE treatments, the mean RI and Cobb angle started to decrease to 2.16 and $52^{\circ}$. In October 2013, after a total of twelve $2-3 \mathrm{hr}$ clinic visits and 12 months of a daily 1.5-2hr Schroth method SSPE, the mean RI decreased to 1.665 and the Cobb angle to $42^{\circ}$. These mean RI and Cobb angle measurements show progression from 2011-2012 and improvement from 2012-2013 as a result of daily SSPE. The authors concluded that the RI can be used as an additional objective measure to show RH improvement with SSPE [10].

\section{Assessment of brace treatment}

The RI was used to assess the initial correction of the $\mathrm{RH}$ in AIS treated with the Dynamic Derotation Brace (DDB). It is well established that scoliotic children and their parents are very much concerned about their trunk deformity (TD). One of their TD components is the RH, which is mainly the expression of the rib deformity. The brace treatment aims not only to hold or correct the central axis, the spinal deformity, but also the TD in the thorax, the RH.

Twenty children with right thoracic $(\mathrm{n}=14)$ and double curves $(n=6)$ (right thoracic left lumbar) were assessed. The SRS/SOSORT inclusion criteria for brace treatment were used, [11-14]. The reference vertebra from which the RI was assessed was documented. Statistical analysis was done using the t-test. The mean thoracic Cobb angle was 27,5 degrees. The posterior margin of the reference vertebra was the T8 in 4 scoliotics, T9 in 2, T10 in 4, T11 in 6, L1 in 2 and L2 in 2, respectively. The mean pre-brace treatment RI was 1,864 and the early post-brace 1,205 respectively, $p=0,007$. It was concluded that the DDB significantly improves the $\mathrm{RH}$ deformity during the initial treatment period in the thoracic curves and in the thoracic component of the double scoliotic curves (Figure 9), [15,16].

\section{Pre-and Postoperative assessment}

The assessment of the post-operative rib-cage deformity correction on the transverse plane, using various surgical techniques, was recently reported [17-20].

The first publication of a follow-up study on surgically treated patient, in which the RI was applied to assess rib hump correction, was by A. Crawford and his group in 2012 [17]. It was initially used in a multi-center study for AIS of Lenke type I curves treated with posterior spinal fusion with or without costoplasty, instrumented with pedicle screws or hybrid constructs, with a minimum follow-up of 2 years. The first group (Group I) was treated with pedicle screws, direct vertebral rotation and no costoplasty, while the second group (Group II) was treated with pedicle screws, vertebral rotation and costoplasty. The RI calculated from the DRCS was measured radiographically and compared between the two groups. The statistical analysis of pre-operative and post-operative $\mathrm{RH}$

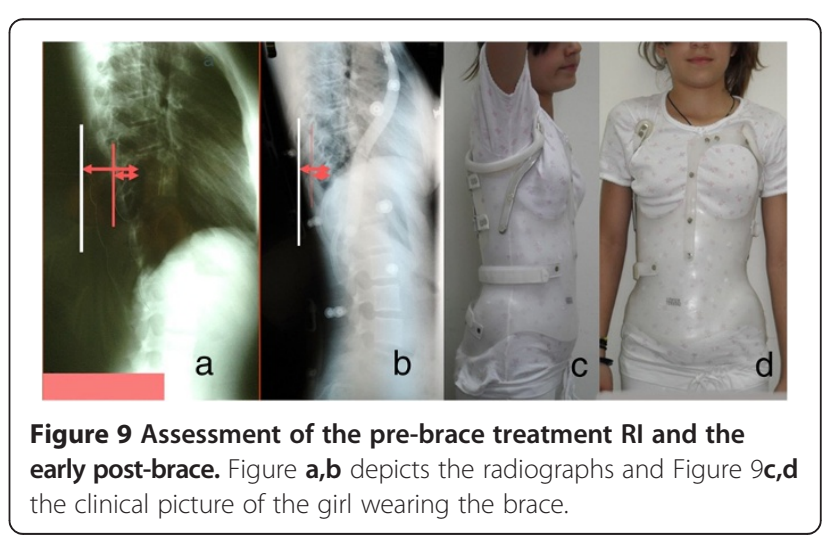


deformity correction using the RI showed that costoplasty combined with pedicle screws and vertebral derotation may significantly improve rib hump deformity, as opposed to pedicle screws and vertebral derotation alone, [17].

Subsequently Stavropoulos et al 2014 measured the RH deformity correction in AIS treated with full screw or hybrid constructs using the DRCS and RI. Sixteen scoliotics (group A) were operated upon using full pedicle screw with median age 15 years and 9 scoliotics (group B) were operated using hybrid construct with median age 17.2 years. RI correction was calculated by subtracting the postoperative RI from the preoperative RI. In group A the mean pre-operative RI was 1.93 and the post-operative 1.37 ( $p<0.001)$. In group B the mean pre-operative RI was 2.06 , while the mean post-operative RI was $1.5(\mathrm{p}=0.008)$. However, between group A and B the RI correction means was not found to be statistically significant, $\mathrm{p}=0.803$. The authors concluded that the $\mathrm{RH}$ deformity correction is the same, no mater what type of spinal construct is used. They also stated that, according to their results, it is implied that the $\mathrm{RH}$ deformity more likely results from asymmetric rib growth rather than from vertebral rotation $[18,19]$.

Haber et al also used the RI, in an effort to provide some more insight on the continuing debate whether all-screw constructs outperform hybrid constructs in small, flexible thoracic AIS. They endorse the statement that "The RI has also been shown to be a reliable method to judge rib rotation owing to its simplicity and ability to be measured on lateral film" [20].

\section{The use of RI and implications for screening policies}

The introduction of the RI resulted in a very important knowledge derived from the statistical analysis of the correlation of Cobb angle to the RI in radiographs from the screening programs referrals.

Approximately $30 \%$ of younger referred girls, aged equal or less than 13 years old with an ATR $\geq 7^{\circ}$, were found to have either a straight spine or a spinal curve under $10^{\circ}$. In this age group the correlation between thoracic deformity in terms of $\mathrm{RH}$ (the transverse plane thoracic deformity) assessed using the RI and the radiographic spinal measurement in terms of Cobb angle, is not statistically significant, while in older referred girls, aged 14-18 years old, it is [21]. Thus, all younger individuals, referred to scoliosis clinics from screening programs, who are identified with a surface deformity (RH) but without a severe scoliotic curve (Cobb angle), are at risk to develop IS. All these children should be followed and not discharged from regular follow-up, see Figure 10. In line with this statement are the Nissinen et al 1993 school screening longitudinal study findings [9].

Without the aforementioned screening program that included younger children in addition to the usual age ranges, the above statement would not be made [22]. The aim of school screening is to identify most or all the individuals with unrecognized IS at an early stage, when a less invasive treatment is more effective. It was earlier emphasized that a further contribution of the screening programs is in the research of IS epidemiology, natural history and aetiology [22]. In addition, these programs are a unique tool for research of IS in humans. In most published articles all aetiopathogenetic factors are studied in animals and not in humans, in contrast with screening programs where any knowledge on IS aetiology is derived from humans, in other words it is human evidenced based knowledge [23]. Such contribution is beyond the original aim of school screening; however

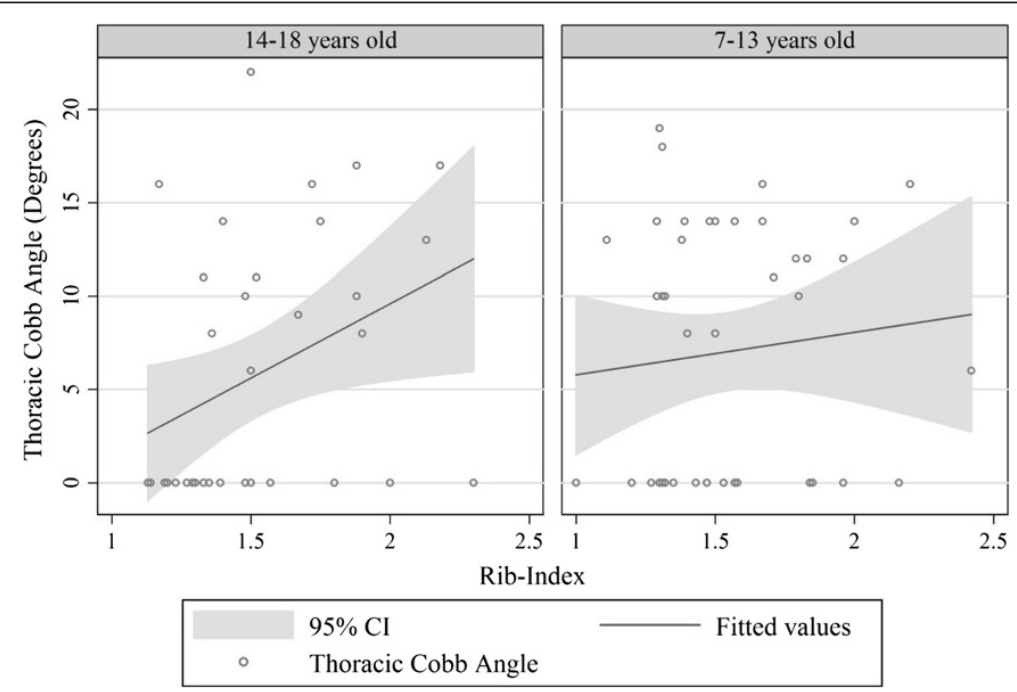

Figure 10 The linear relationship between thoracic Cobb angle and RI is graphically depicted. There is only linear association between thoracic Cobb Angle and rib-index in the age group of 14-18 years. (Predicted Thoracic Cobb Angle $=-6.357+7.974$ X (Rib-Index) [16] . 
it is very important because we can expand our knowledge and adequately understand the pathogenesis of IS. The role of biological factors such as the menarche, the lateralization of the brain, the handedness, the thoracic cage, the intervertebral disc, the melatonin secretion, as well as the role of environmental factors such as the light and the impact of the geographical latitude in IS prevalence, were studied in children referred from school screening [22]. The current evidence supports that school screening programs should be continued not only for early detection of IS but also as a basis for epidemiological surveys until we learn much more about the aetiology of IS.

The role and usefulness of RI in the school screening program, at a first consideration, seems to be minor in the screening program itself, but very important for research purposes. However, the introduction of RI and its correlation to the Cobb angle of the radiographs of the children referred from school screening, shaped the age range of them who must be followed, so that our decision on this issue was more thorough. Therefore the importance of RI in the school screening program is significant.

\section{Reference of the RI method in spinal textbooks}

A safe way to recognize a method's importance is to notice if it is referred in pertinent classical text books. The RI method used to assess the RH, in other words the transverse plane thoracic deformity, is currently referred in some classical spinal text books [24,25].

\section{Citations in Google Scholar}

For the article - the first publication on DRCS and RI The Double Rib Contour Sign (DRCS) in Lateral Spinal Radiographs. Aetiologic Google Scholar provides 45 references (search on 29/8/2014), see below:

The Double Rib Contour Sign (DRCS) in Lateral Spinal Radiographs. Aetiologic

TB GRIVAS, S Dangas, BD Polyzois... - Research Into Spinal ..., 2002 - books.google.com

Abstract: All lateral spinal radiographs in idiopathic scoliosis show a DRC sign of the thoracic cage, a radiographic expression of the rib hump. The outline of the convex overlies the contour of the concave ribs. The aim of this study is to assess this DRC sign in children.

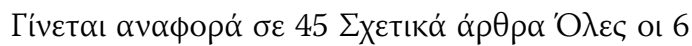
$\varepsilon \kappa \delta$ $\chi \dot{\varepsilon} \varsigma$ П $\alpha \rho \alpha \dot{\theta} \theta \varepsilon \sigma \eta$

\section{Summary}

In this article the DRCS and the RI are analyzed. The history of presentations and the first publication of DRCS-RI are reported. The original source of the study was the referred children for IS from our school screening program; the description and quantification of the DRCS and the RI are explained. The reliability study for RI and how much the RI is affected by the distance between the radiation source and the irradiated individual is another topic of this article. The implications of the DRCS and RI in the aetiology of IS are analyzed. The clinical applications of RI in the assessment of the thoracic deformity in the transverse plane, the outcomes of physiotherapy, bracing and surgical treatment are described. The use of RI and implications for screening policies are also detailed. Finally, the spinal text books referring this method and the Google Scholar citations are enumerated.

\section{Consent}

Written informed consent was obtained from the patient's guardian/parent/next of kin for the publication of this report and any accompanying images.

\section{Competing interests}

The author declares that he has no competing interests.

\section{Authors' contributions}

The author conceived the RI, coined the DRCS, implemented the literature search and drafted the manuscript.

\section{Acknowledgements}

The author would like to express his gratitude to all the colleagues who so far participated to the "Thriasio" and "Tzaneio" General Hospitals School Screening for Scoliosis program, and especially Mrs Christina Mazioti, Health visitor serving in "Thriasio" and "Tzaneio" General Hospitals, for her key role in the implementation of these programs, feeding the collected data derived from the program to SPSS program and taking part in our Scoliosis Clinic.

Received: 28 August 2014 Accepted: 31 October 2014 Published online: 20 November 2014

\section{References}

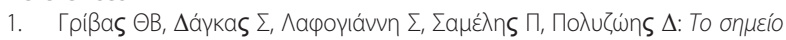

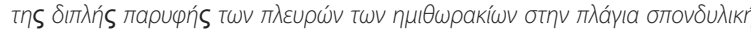

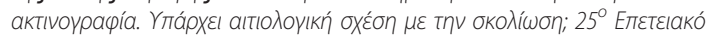

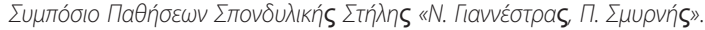
Пátpa: Hotel Porto Rio; 1999.

2. Grivas TB, Daggas S, Polyzois B, Samelis P: The Double Rib Contour Sign $(D R C S)$ in lateral spinal radiographs: aetiologic implications for scoliosis. Clermont, France: International Research Society of Spinal Deformities meeting; 2000. 23-26 May 2000, Proceedings: page 9.

3. Grivas TB, Dangas S, Polyzois BD, Samelis P: The Double Rib Contour Sign (DRCS) in lateral spinal radiographs: aetiologic implications for scoliosis. Stud Health Technol Inform 2002, 88:38-43.

4. American College of Radiology (ACR) - SPR practice guideline for the performance of radiography for scoliosis in children. Revised 2009. http://www. acr.org/ /media/ACR/Documents/PGTS/quidelines/Scoliosis.pdf.

5. Soultanis K, Tsiavos K, Grivas TB, Stavropoulos N, Sakellariou V, Mavrogenis A, Papagelopoulos PJ: Reliability study for the "rib index" in chest radiographs of a control group. $11^{\text {th }}$ International Conference on Conservative Management of Spinal Deformities, $9^{\text {th }}$ SOSORT Annual Meeting, 8-10 May 2014, Wiesbaden (Germany), 6-7 May 2014, Wiesbaden (Germany). Proceedings: page 28.

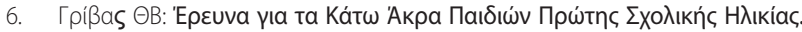

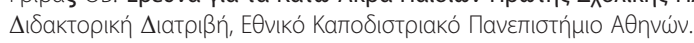
http://hdl.handle.net/10442/hedi/3440 Grivas TB, Survey on the lower limbs of early school age children. PhD Thesis. Degree Grantor National and Kapodistrian University of Athens, Greece. National Archive for PhD Theses http://www.didaktorika.gr/eadd/handle/10442/3440; 1988

7. Grivas TB, Soultanis K, Mazioti C, Kechagias V, Akriotis A, Athanasopoulos K, Naskas C, Tagkas D: The double rib contour sign on lateral spinal 
radiographs: how much the rib index is affected by the distance between the radiation source and the irradiated individual? Oral Presentation at the $11^{\text {th }}$ International Conference on Conservative Management of Spinal Deformities, $9^{\text {th }}$ SOSORT Annual Meeting, 8-10 May 2014, Wiesbaden (Germany), $4^{\text {th }}$ SOSORT Educational Courses, 6-7 May 2014, Wiesbaden (Germany). Publication of the Abstract: Conference proceedings: page 36.

8. Grivas TB, Vasiliadis E, Dangas S: The importance of double rib contour sign in the aetiology of idiopathic scoliosis. Podium Presentation: Annual Meeting of Spinal Experts Group. Thessaloniki Greece: Hyatt Regency Hotel. December 9-10 2005. Abstract Proceedings: page 42; 2005.

9. Nissinen M, Heliövaara M, Seitsamo J, Poussa M: Trunk asymmetry, posture, growth, and risk of scoliosis. A three-year follow-up of Finnish prepubertal school children. Spine (Phila Pa 1976) 1993, 18(1):8-13.

10. Lebel $A$ and Lebel $\mathrm{V}$ : Rib index, an objective measure to document changes of the rib hump deformity in a risser 4 progressive AIS patient treated with the Schroth method. 11th International Conference on Conservative Management of Spinal Deformities, 9th SOSORT Annual Meeting, 8-10 May 2014, Wiesbaden, Germany. Proceedings: page 40

11. Richards BS, Bernstein RM, D'Amato CR, Thompson GH: Standardization of criteria for adolescent idiopathic scoliosis brace studies: SRS Committee on Bracing and Nonoperative Management. Spine (Phila Pa 1976) 2005, 30(18):2068-2075. discussion 2076-7. Review.

12. Weiss H-R, Negrini S, Rigo M, Kotwicki T, Hawes M, Grivas T, Maruyama T, Landauer F, committee Sg: Indications for conservative management of scoliosis (guidelines). Scoliosis 2006, 1(1):5.

13. Negrini S, Aulisa AG, Aulisa L, Circo AB, de Mauroy JC, Durmala J, Grivas TB, Knott P, Kotwicki T, Maruyama T, Minozzi S, O'Brien JP, Papadopoulos D, Rigo M, Rivard CH, Romano M, Wynne JH, Villagrasa M, Weiss HR: Zaina F2011 SOSORT guidelines: Orthopaedic and Rehabilitation treatment of idiopathic scoliosis during growth. Scoliosis 2012, 7(1):3. doi:10.1186/1748-7161-7-3.

14. Negrini S, Grivas TB, Kotwicki T, Rigo M, Zaina F: International Society on Scoliosis Orthopaedic and Rehabilitation Treatment (SOSORT) Guidelines on "Standards of management of idiopathic scoliosis with corrective braces in everyday clinics and in clinical research": SOSORT Consensus 2008. Scoliosis 2009, 4:2. doi:10.1186/1748-7161-4-2.

15. Grivas TB, Triantafyllopoulos G, Mazioti C: Assessment of early RH deformity correction in adolescent idiopathic scoliosis treated with a dynamic derotation brace using the double rib contour sign. Scoliosis 2013, 8(Suppl 2):054

16. Grivas TB: Chapter: Radiological appraisal of thoracic deformity improvement or deterioration using the convex/concave rib-hump index ('double rib contour sign') in curves Lenke Type 1, 3, 5 and 6. In Scoliosis Research Society - Half-Day Courses. Scoliosis Research Society, Editors: SRS 2012-2013 Education Committee; 29-30.

17. Lykissas MG, Sharma V, Crawford AH: Assessment of RH Deformity Correction in Adolescent Idiopathic Scoliosis With or Without Costoplasty Using the Double Rib Contour Sign. J Spinal Disord Tech 2012, [Epub ahead of print].

18. Stavropoulos NA, Soultanis KC, Grivas TB, Starantzis KA, Tsiavos K, Sarlikiotis T, Mimidis G, Papagelopoulos PJ: Measurement of rib hump deformity correction in adolescent idiopathic scoliosis treated with full screw or hybrid constructs using the double rib contour sign. 7o Panhellenic Spinal Meeting, 31.10 - 2.11 2013. Makedonia Palace, Thessaloniki, Greece.

19. Stavropoulos NA, Soultanis KC, Grivas TB, Tsiavos K, Starantzis K, Sarlikiotis T, Karamanis E, Papagelopoulos PJ: Measurement of RH deformity correction in adolescent idiopathic scoliosis treated with full screw or hybrid constructs using the double rib contour sign. London, United Kingdom: 15th EFORT Congress; 2014.

20. Haber LL, Hughes JD, Womack ED, Roberson RM, Wrigh PB: Screw versus hybrid constructs for flexible thoracic curves in adolescent idiopathic scoliosis: a prospective, randomized study. Spine Deform 2014, 2(5):367-373.

21. Grivas TB, Vasiliadis ES, Mihas C, Savvidou O: The effect of growth on the correlation between the spinal and rib cage deformity: implications on idiopathic scoliosis pathogenesis. Scoliosis 2007, 2:11.

22. Grivas TB, Vasiliadis E, Savvidou OD, Triantafyllopoulos G: What a school screening program could contribute in clinical research of idiopathic scoliosis aetiology. Disabil Rehabil 2008, 30(10):752-762. doi:10.1080/09638280802041086
23. Grivas TB, Vasiliadis ES, Kaspiris A, Triantafyllopoulos G: Human Evidenced Based Study on Idiopathic Scoliosis Aetiology. Orthopaedic Proceedings July 2011. J Bone Joint Surg Br 2011, 93-B(III):368. http://www.bjjprocs. boneandjoint.org.uk/content/93-B/SUPP_III/368.1.

24. Kim DH, Betz RR, Huhn SL, Newton PO: Surgery of the Pediatric Spine. First Publication 2008. 333 Seventh Ave., New York: Thieme Medical Publishers, Inc. NY 10001, ISBN (Americas): 9781588903426, ISBN (EUR, Asia, Africa, AUS): 9783131419316, page 809.

25. Newton PO, O'Brien MF, Shufflebarger HL, Betz R, Dickson RA, Harms J: Idiopathic scoliosis: The Harms Study Group treatment guide. 1st edition. In chapter 5, by Alvin H. Crawford, Alan E. Oestreich, Linda P. D'Andrea, Joshua H. Heller, and Patrick Cahill, ISBN (Americas): 9781604060249; 2010.

doi:10.1186/s13013-014-0020-9

Cite this article as: Grivas: Rib index. Scoliosis 2014 9:20

\section{Submit your next manuscript to BioMed Central and take full advantage of:}

- Convenient online submission

- Thorough peer review

- No space constraints or color figure charges

- Immediate publication on acceptance

- Inclusion in PubMed, CAS, Scopus and Google Scholar

- Research which is freely available for redistribution

Submit your manuscript at www.biomedcentral.com/submit
C) Biomed Central 